\title{
Competencia digital de estudiantes universitarios para el aprendizaje del inglés en tiempos de la COVID-19
}

\author{
Digital competence of University Students in English Language Learning \\ during COVID-19 times
}

\author{
Uriel Ruiz-Zamora $^{a}$
}

\begin{abstract}
:
Nowadays, English Language Teaching uses technology as a necessary tool in the teaching-learning process in order it becomes more effective. This article presents the results of the research made about the use of Information and Communication Technologies (ICT) as a digital competence for English language self-learning by university students during the COVID-19 pandemic. The methodology of this study is quantitative descriptive; 85 students of the Faculty of Languages UAEMex were selected by a nonprobabilistic sampling. The method of collecting data was with the Background Questionnaire (Liu, et. al, 2008) to participants, which was designed and administered electronically. The results suggest that despite students who are indeed using their digital competences for English language self-learning, they are having some restrictions due to their internet connection. Finally, it is established that this health contingency raises challenges for the English Language teachers as not to depend only on the Internet connection for student's self-learning.
\end{abstract}

\section{Keywords:}

Digital Competence, English Language Learning, COVID-19, University Students

\section{Resumen:}

La enseñanza del inglés en la época actual se vale del uso de tecnologías como herramientas necesarias en el proceso de enseñanza - aprendizaje del idioma para que éste sea más efectivo. El artículo presenta los resultados de la investigación realizada sobre el empleo de las TIC como competencia digital para el aprendizaje autónomo de la lengua inglesa por estudiantes universitarios durante la pandemia del Coronavirus COVID-19. La metodología del estudio es cuantitativa descriptiva; los participantes fueron 85 estudiantes de la Facultad de Lenguas UAEMex seleccionados mediante un muestreo no probabilístico. La obtención de datos fue a través del Background Questionnaire (Liu, et. al, 2008), el cual fue diseñando y administrado vía electrónica. Los resultados sugieren que los estudiantes sí han hecho uso de sus competencias digitales para el autoaprendizaje de la lengua inglesa con algunas limitantes por la conectividad a Internet. Finalmente, se establece que esta contingencia sanitaria plantea retos para los profesores para no depender únicamente de la conectividad a Internet para el estudio autónomo.

\section{Palabras Clave:}

Competencia digital, aprendizaje del inglés, COVID-19, estudiantes universitarios

\section{INTRODUCTION}

La educación del siglo XXI contempla el uso de nuevas tecnologías como estrategia para la transmisión de conocimientos, principalmente a las Tecnologías de la Información y la Comunicación (TIC). Dichas tecnologías son integradas en el proceso de enseñanza-aprendizaje de lenguas, en este caso particular para la lengua inglesa, la cual

Corresponding author, Universidad Autónoma del Estado de México, https://orcid.org/0000-0002-2691-5490, Email: uruizz76@gmail.com 
hace uso de las TIC en su metodología, métodos y técnicas por su naturaleza multidimensional. Las TIC son recursos que hacen el proceso de enseñanza-aprendizaje del inglés más efectivo (Vasan, 2020).

La incorporación de las TIC en el aprendizaje del inglés en el presente siglo demanda, por parte de los estudiantes, el desarrollo de la competencia digital, la cual se relaciona con las habilidades computacionales de acuerdo con Morze, Smyrnova-Trybulska, y Boiko (2019). Competencia que se volvió fundamental para el aprendizaje durante el primer semestre del 2020 debido a la contingencia sanitaria por la aparición de la enfermedad por coronavirus (COVID-19) (Organización Mundial de la Salud, 2020).

Dicha enfermedad, provocó que la Secretaría de Educación Pública de México (SEP) estableciera que el receso escolar comprendería del lunes 23 marzo hasta el 20 de abril del 2020 (SEP, 2020); sin embargo, el receso escolar se ha extendido hasta finalizar el periodo escolar 2019 - 2020. La decisión de la SEP fue tomada en cuenta por todas las instituciones educativas a nivel nacional, incluyendo a la Universidad Autónoma del Estado de México (UAEMex), quien, a través de su Rector, Dr. Alfredo Barrera Baca, anunció la suspensión de las clases presenciales a partir del 19 de marzo de 2020 en congruencia con el Gobierno de México (Barrera , 2019).

La suspensión de las clases presenciales en la UAEMex hizo que, tanto estudiantes como profesores, adaptaran sus cursos a la modalidad virtual. En el caso específico de las clases de inglés, se desconoce si los estudiantes universitarios hacen uso de manera autónoma del internet como TIC para el aprendizaje de la lengua inglesa en tiempos de la COVID-19. Por lo tanto, se pretende Determinar si los estudiantes universitarios usan el internet para el autoaprendizaje de la lengua inglesa durante la pandemia de la COVID-19, independientemente de las actividades programadas por los profesores.

¿Qué tipo de dispositivo electrónico tienen los estudiantes para acceder a Internet?, ¿Cuánto tiempo utilizan el Internet en tiempos de la COVID-19?, ¿Qué tipo de contenidos acceden en Internet para estudiar inglés?, ¿Ha sido de utilid ad el empleo del Internet para estudiar inglés durante la suspensión de clases presenciales?

La investigación permitirá conocer de manera exploratoria el acceso que tienen los estudiantes universitarios al Internet en sus lugares de residencia y cómo lo utilizan para el aprendizaje de la lengua inglesa durante la etapa de cuarentena por la COVID-19.

\section{COMPETENCIA DIGITAL Y LA LENGUA INGLESA}

La educación actual ha evolucionado de gran manera, lo que tiene como consecuencia el no seguir enseñando $y$ aprendiendo de igual manera que se realizó en el siglo XX. En este sentido Prensky (2011, p. 111) explica que "Una de las grandes diferencias entre enseñar en el siglo XXI y en el pasado es que en el pasado las cosas no cambiaban muy deprisa". El proceso educativo actual no puede dejar de lado el uso de las TIC como herramientas esenciales para obtener y compartir información entre sus involucrados.

Las TIC son definidas por Cabero (2015) como "Recursos percibidos como facilitadores y transmisores de información y recursos educativos para los estudiantes, que pueden ser adaptados a las necesidades y características propias de los sujetos, pudiendo conseguir con ellos una verdadera formación audiovisual, multimedia e hipertextual" (p. 22).

Además, de la obtención y transferencia de la información entre los usuarios, éstos tienen la capacidad de modificar los contenidos para adaptarlos a su propio contexto, y así obtener el máximo provecho de los mismo. Es decir, los estudiantes, de manera particular, se vuelven en constructores de sus propios sus conocimientos. Asimismo, las TIC son aquellos dispositivos tecnológicos que permiten la comunicación y colaboración entre personas para la generación, intercambio, difusión, gestión y acceso al conocimiento (Cobo, 2009; Tello, 2011).

La información que se crea y se transmite por medio de las TIC puede tener diversos formatos, como pueden ser datos textuales o de voz, imágenes, elementos de multimedia entre otros (Tello, 2011). El dominio, por parte de los usuarios, de las distintas actividades que se pueden realizar con las TIC hace referencia a la literacidad digital. Esta literacidad incluye competencias tales como literacidad computacional, literacidad TIC, literacidad de la información y literacidad multimedia (UNESCO, 2018).

La literacidad digital empleada por la sociedad en sus diversas actividades, tanto personales como profesionales, demuestra su competencia digital, la cual evidencia el uso de una diversidad de tecnologías de la información y la comunicación para resolver problemáticas en su vida.

La competencia digital está considerada como una de las ocho competencias clave para el aprendizaje a lo largo de la vida del Marco Europeo de Referencia (Key Competences For Lifelong Learning - A European Reference Framework). La competencia digital involucra el uso fehaciente y crítico de las Tecnologías de la Sociedad de la Información para el trabajo, diversión y comunicación; sumando de habilidades tecnológicas como el uso de la computadora para la búsqueda, evaluación, almacenamiento, producción e intercambio de información; además de comunicarse y participar colaborativamente por medio del internet (OJEU, 2006).

El empleo de las TIC en el proceso de enseñanza de lenguas es un aspecto que ha ido en aumento en la última década. Los profesores utilizan distintas tecnologías para hacer llegar el conocimiento a los estudiantes, quienes, de una manera más 
familiarizada al ser nativos digitales, emplean dichas tecnologías para decodificar los contenidos temáticos por medio de la tecnología empleada demostrando su competencia digital.

Lo anterior plantea un reto para los profesores quienes deben actualizar sus conocimientos digitales para poder integrarse a las nuevas formas de enseñanza. Ferrari (2013) explica que la literacidad digital es una de las competencias que los docentes deberán dominar para continuar con su profesión en el siglo XXI. Sin embargo, la competencia digital no debe ser dominada únicamente por los profesores, sino que los estudiantes también deben desarrollar la misma para lograr un mayor aprendizaje.

Particularmente, el dominio de las TIC por parte de los estudiantes universitarios presupone una mayor competencia digital al ser nativos digitales, ya que como menciona Chandrasena (2019), los estudiantes hacen uso de su literacidad digital por medio de las computadoras, multimedia, teléfonos celulares, tecnologías que les son familiares en sus días.

El uso apropiado de las TIC, por parte de los estudiantes, promueve el aprendizaje de las distintas habilidades de lenguas. Los estudiantes deben ser capaces de buscar actividades que apoyen significativamente lo estudiando en las clases de lengua. En este sentido, los profesores deben promover el uso de las TIC entre sus estudiantes, proporcionándoles la instrucción y guía para la búsqueda de información que de calidad para el aprendizaje de lenguas.

Jeremy Harmer (2015) expone que, una de las tareas más importantes de los maestros es motivar a los estudiantes a utilizar recursos materiales y tecnológicos por ellos mismos, para así convertirse más independientes en su aprendizaje. El aprendizaje por medio de las tecnologías permite a los estudiantes controlar su propio avance, así como tener acceso a mayor información de la cual el docente no tiene control (Pourhosein Gilakjani \& Sabouri, 2014).

La guía de los profesores, en relación con el uso y empleo de las TIC, para el estudio autónomo de la lengua inglesa es fundamental para que los estudiantes logren una competencia digital, así como el dominio de la lengua inglesa, promoviendo cada vez más el estudio propio no formal por parte de los discentes en este siglo XXI. La guía proporcionada a los discentes, por parte de los docentes, es esencial para el aprendizaje, ya que, de acuerdo con Bennett, Maton, \& Kervin (2008) los estudiantes no siempre tienen las habilidades tecnológicas a pesar de haber nacido en un mund o de alta tecnología.

\section{CORONAVIRUS COVID-19}

Las actividades cotidianas de la humanidad se vieron modificadas por la aparición de la COVID-19 a finales del año 2019, y que se ha extendido durante el año 2020, ésta es una enfermedad causada por un nuevo coronavirus recientemente descubierto.

La Organización Mundial de la Salud (2020) expone que, el 31 de diciembre del 2019 las autoridades de China le informaron la aparición de casos de neumonía en la ciudad de Wuhan, desconociendo sus causas. Los coronavirus son virus que causan desde gripes comunes hasta enfermedades más graves. La COVID-19, así denominado, es un coronavirus que no se había presentado en los seres humanos. El Centro de Coordinación de Alertas y Emergencias Sanitarias (CCAES) del Ministerio de Sanidad del Gobierno de España, describe a los coronavirus:

Los coronavirus son miembros de la subfamilia Orthocoronavirinae dentro de la familia Coronaviridae (orden Nidovirales) (11). Esta subfamilia comprende cuatro géneros: Alphacoronavirus, Betacoronavirus, Gammacoronavirus y Deltacoronavirus de acuerdo a su estructura genética... Hasta la aparición del SARS CoV-2, se habían descrito seis coronavirus en seres humanos ... El coronavirus (12) SARS-CoV-2 supone el séptimo coronavirus aislado y caracterizado capaz de provocar infecciones en humanos (CCAES, 2020).

A partir de enero de 2020, la humanidad estuvo al pendiente del comportamiento de la COVID-19 en China y la actuación de sus autoridades. El virus se esparció rápidamente por el mundo, lo que llevó a la OMS a establecerlo como una pandemia al detectarse en 114 países en el mes de marzo 2020. La OMS reportó que más del $40 \%$ de los casos se registraba en Europa en marzo 2020, lo que la establecía como el epicentro de la pandemia. El porcentaje de mortalidad se elevó al 63\% en el mes de abril 2020 (Organización Mundial de la Salud, 2020).

En el continente americano el primer caso de la COVID-19 se reportó Estados Unidos el 20 de enero del 2020 y en América Latina el primer caso se detectó en el mes de febrero 2020. En el continente americano se ha detectado el virus en 54 países y regiones (Organización Panamericana de la Salud, 2020).

\section{METODOLOGIA}

La investigación se llevó a cabo en la Licenciatura en Lenguas (LLE) de la Facultad de Lenguas de la UAEMex, en la Ciudad de Toluca, Estado de México; en el mes de junio 2020, durante el periodo de confinamiento por la COVID-19. El curriculum de la LLE (UAEM, 2009) contempla ocho niveles obligatorios de lengua inglesa para obtener las competencias del nivel ALTE 4 (Association of Language Testers in Europe) equivalente al nivel C1 del Marco Común Europeo de Referencia (MCER). El nivel C1 es definido como usuario 
competente, capaz de comunicarse con propiedad, sensibilidad y la habilidad para tratar temas poco familiares (ALTE, 2018).

Actualmente, no se tiene conocimiento sobre el dominio y uso de las TIC, que tienen los estudiantes de la LLE para realizar el estudio de la lengua inglesa de manera autónoma en los tiempos de la COVID-19; además se desconoce los medios tecnológicos con los que cuentan los alumnos para realizar sus actividades de estudio a distancia y autónoma.

La investigación se realizó desde la perspectiva cuantitativa descriptiva con el objetivo de determinar el uso que se hace del Internet para el autoaprendizaje de la lengua inglesa por parte de los estudiantes de la LLE durante la pandemia del COVID-19. La obtención de los datos se llevó a cabo por medio del cuestionario Background Questionnaire (Liu, et. al, 2008), el cual fue adaptado, y se realizó una ronda con expertos para calificar la pertinencia y claridad de las preguntas. El cuestionario incluye tres secciones: a) Información personal, b) Nivel y conocimiento computacional, y c) Aprendizaje del inglés con el uso de la web. Este fue diseñado en Microsoft Forms y distribido via correo electrónico.

Un total de 85 estudiantes de la LLE de la Facultad de Lenguas de distintos semestres participaron en la investigación; se les solicitó responder el cuestionario de manera voluntaria, además, se les hizo saber que el cuestionario no tendría ninguna repercusión en sus calificaciones de la asignatura Lengua inglesa.

\section{RESULTADOS Y DISCUSION}

\section{Sección a) Información personal}

Las respuestas obtenidas en el cuestionario muestran que el $62 \%$ de los participantes tiene entre 18 y 19 años, mientras que únicamente el $6 \%$ tiene más de 25 años (Gráfica 1). El $80 \%$ es de género femenino y el $20 \%$ de género masculino.

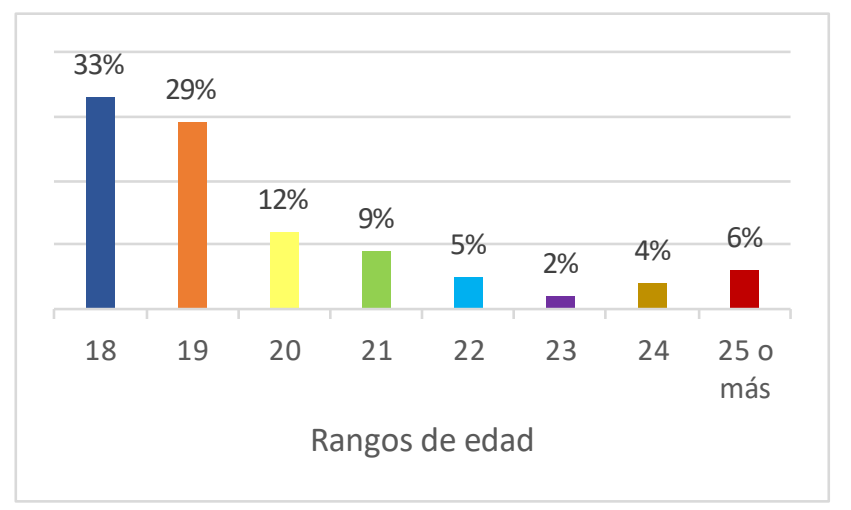

Gráfica 1. Edades de los estudiantes.

\section{Sección b) Nivel y conocimiento computacional}

La herramienta tecnológica más utilizada por los estudiantes para realizar sus tareas y actividades de estudio autónomo es el teléfono celular; seguido de la Laptop. La usabilidad del teléfono celular permite a los estudiantes acceder a los contenidos establecidos por los maestros, así como para el autoaprendizaje de una forma más práctica. La herramienta menos utilizada es la Tablet a pesar de ser una herramienta de alta usabilidad (Gráfica 2).

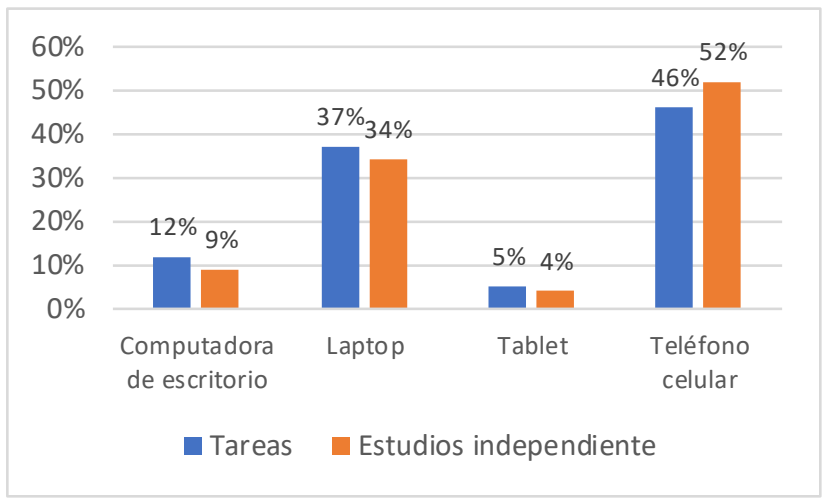

Gráfica 2. Tecnologías para tareas y estudio autónomo.

La gran mayoría de los estudiantes (89\%) tiene conexión propia a Internet durante la COVID-19, ya sea por proveedor de servicio casa o por el uso de datos del teléfono celular; lo que les permite realizar sus actividades académicas establecidas en las clases, así como las de estudio autónomo en su hogar. Una minoría de alumnos que no tiene acceso a Internet en casa, lo que puede complicarles la realización de las tareas académicas formales y en autonomía (Gráfica 3).

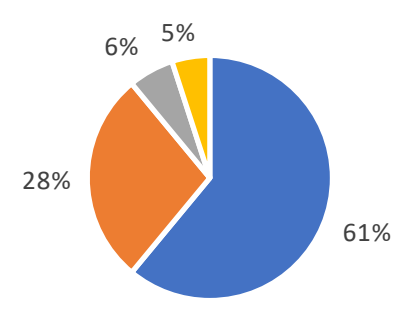

- Proveedor de servicio en casa

- Datos del teléfono celular

- Cibercafé

- Otras

Gráfica 3. Medio de conexión a internet durante la COVID19.

El $47 \%$ de los estudiantes utiliza el Internet entre 8 horas o más a lo largo de la pandemia COVID-19; mientras que $44 \%$ de estudiantes utiliza el internet entre 4 y 7 horas. De este $91 \%$ de estudiantes, el $86 \%$ tiene conexión por proveedor de servicio en casa o por el uso de datos del teléfono celular, el $5 \%$ restante utiliza fichas con internet y datos móviles (Gráfica 4). 


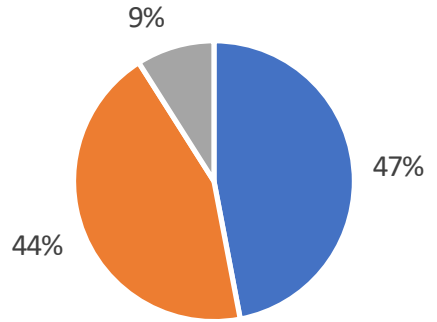

- 8 o más horas de 4 a 7 horas de 2 a 4 horas

Gráfica 4. Horas de uso de Internet durante COVID-19.

En la pregunta, ¿Normalmente para que utilizas el Internet durante la pandemia COVID-19?, se les pidió a los estudiantes que seleccionaran distintas actividades. Las tareas académicas como es el envío de trabajos, el estudio, la revisión del correo y la investigación son las más realizadas. Mientras que, entre las actividades de ocio, el entretenimiento es la más practicada; el revisar las noticias y comprar no llevadas a cabo por una gran cantidad de estudiantes (Gráfica $5)$.

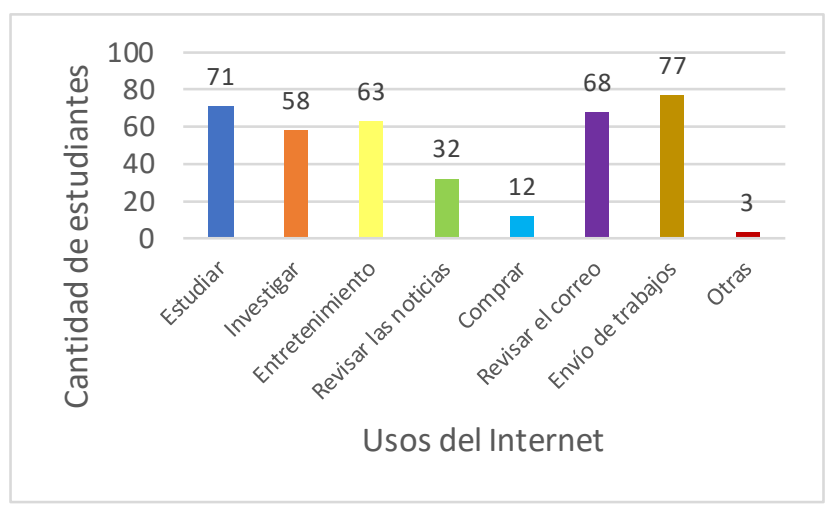

Gráfica 5. Usos del Internet en la pandemia COVID-19.

El uso de la computadora muestra que solamente un estudiante tiene menos de dos años utilizando esta herramienta en su vida, lo que demuestra que su contacto con dicha tecnología fue hasta su ingreso a la universidad. El mayor porcentaje de uso de la computadora tiene entre 5 y 10 años, con el 39\%. El empleo del teléfono inteligente es muy similar entre los participantes, el mayor rango de su utilización es entre 5 y 10 años, con el $51 \%$ mientras que el $4 \%$ tiene menos de dos años de utilizar el teléfono inteligente como herramienta tecnológica. (Gráfica 6).

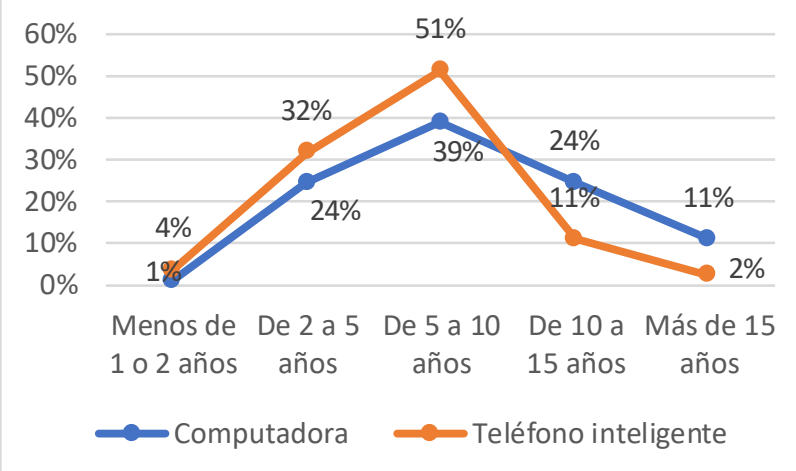

Gráfica 6. Años de uso de las tecnologías.

Los años de utilización de la computadora reflejan un excelente dominio de dicha tecnología por la mitad de los estudiantes, mientras que el $40 \%$ se considera mediantemente experimentado en el manejo de esta tecnología. El estudiante que tiene menos de dos años utilizando la computadora se dice poco experimentado en el uso de la misma; sin embargo, este mismo estudiante se dice experimentado en el manejo del teléfono celular, supliendo la ausencia del manejo de la computadora.

Un gran número de estudiantes, quienes en su mayoría pertenecen a la generación $\mathrm{Z}$, expresan tener dominio muy experimentado o experimentado del teléfono inteligente, sumando el 83\%; este dominio tiene correspondencia al ser el teléfono inteligente, una tecnología propia de su época. En contraste, uno de los estudiantes mayores de 25 años, se dice nada experimentado en el manejo del teléfono inteligente (Gráfica 7).

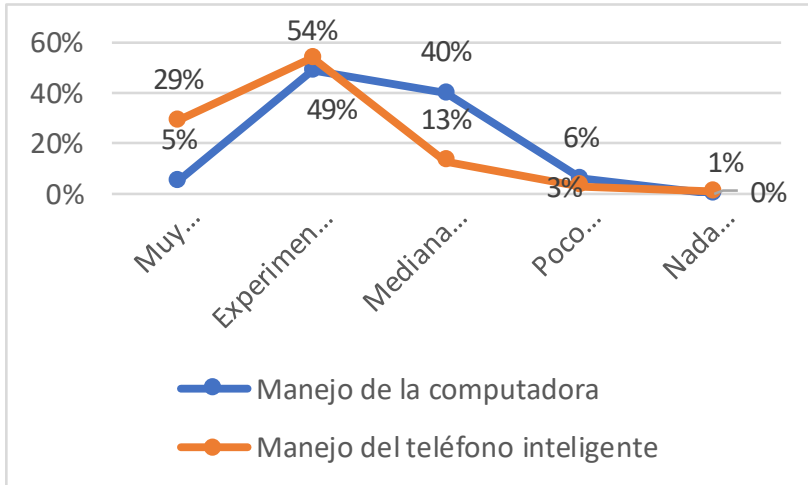

Gráfica 7. Experiencia en manejo de la tecnología.

La experiencia en el manejo de las TIC evidencia la existencia de la literacidad digital en los estudiantes. Sin embargo, parte esencial de la literacidad digital estable la búsqueda, evaluación, almacenamiento, producción e intercambio de información; además de comunicarse y participar colaborativamente por medio del internet, logrando así la competencia digital (OJEU, 2006).

En este sentido, un pequeño grupo de estudiantes se consid era muy experimentado en el uso del Internet para la búsqueda de 
información, la gran mayoría se considera experimentado (55\%) y un poco más del $30 \%$ considera ser medianamente experimentado. Ningún alumno expresó no tener nada de experiencia en la búsqueda de información en la Web.

La relación entre el manejo de la computadora y el teléfono inteligente es proporcional al dominio de la búsqueda de información en la Web, donde el mayor porcentaje de estudiantes se encuentra en el nivel experimentado (Gráfica 8).

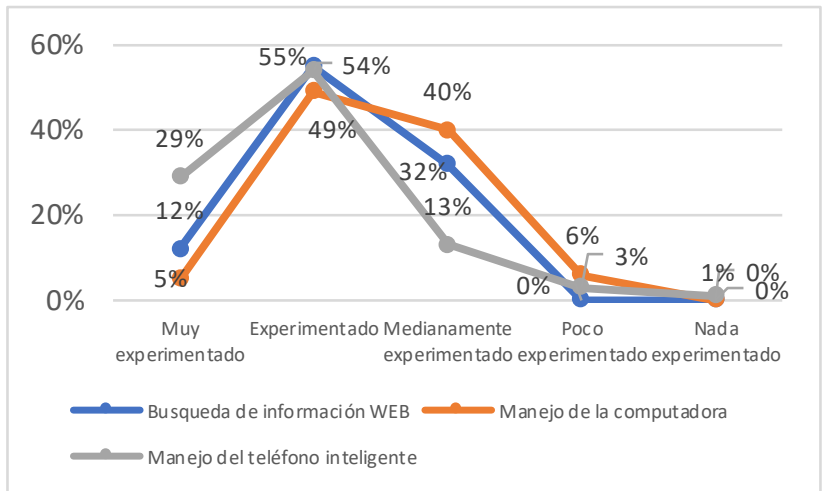

Gráfica 8. Manejo de las TIC y búsqueda de información.

\section{Sección c) Aprendizaje del inglés con el uso de la web}

Primeramente, los estudiantes indicaron si han utilizado el Internet para estudiar inglés de manera autónoma durante la pandemia COVID-19. El $73 \%$ indicó sí estar estudiando de manera autónoma durante la cuarentena (las preguntas subsecuentes fueron respondidas únicamente por este grupo de estudiantes).

Este grupo de alumnos indicó haber estado estudiando todas las habilidades de la lengua inglesa y la gramática, además de otros aspectos como vocabulario en inglés. La habilidad más estudiada en autonomía es la comprensión oral, seguida por la gramática. La habilidad menos estudiada es la producción escrita.

El tiempo empleado por más de la mitad de los estudiantes para el estudio autónomo de la lengua inglesa en la COVID19, es entre dos y cuatro horas a la semana. Mientras que únicamente el $8 \%$ estudia ocho horas o más a la semana. Los sitios y medios que utilizan los alumnos para realizar estudio autónomo se ordenan en tres categorías a) Sitios libres para aprender inglés, b) Cursos de inglés con costo y c) Sitios y medios no específicos para estudiar inglés (Tabla 1).

\begin{tabular}{|c|c|c|}
\hline $\begin{array}{l}\text { Sitios libres para } \\
\text { aprender inglés }\end{array}$ & $\begin{array}{l}\text { Cursos de inglés } \\
\text { con costo }\end{array}$ & $\begin{array}{c}\text { Sitios y medios no } \\
\text { específicos para } \\
\text { estudiar inglés }\end{array}$ \\
\hline $\begin{array}{l}\text { - } \quad \text { BBC learning } \\
\text { English } \\
\text { - } \quad \text { Cake - Learn }\end{array}$ & $\begin{array}{ll}\text { - } & \text { Babbel } \\
\text { - } & \text { Coursera } \\
\text { - } & \text { Edx learn }\end{array}$ & $\begin{array}{ll}\text { - } & \text { Bored panda } \\
\text { - } & \text { Daily news } \\
\text { - } & \text { Música }\end{array}$ \\
\hline
\end{tabular}

\begin{tabular}{|c|c|c|}
\hline $\begin{array}{ll} & \text { English for } \\
& \text { Free } \\
\text { - } & \text { Cambrige } \\
\text { dictionary } \\
\text { - } & \text { Duolingo } \\
\text { - } & \text { Englisch- } \\
\text { hilfen } \\
\text { - } & \text { ESLeschool } \\
\text { - } & \text { HelloTalk } \\
\text { - } & \text { Learnenglish. } \\
& \text { britishcouncil } \\
\text { - } & \text { Linguee } \\
\text { - } & \text { Luvlingua } \\
\text { - } & \text { Oxfordonline } \\
\text { english } & \text { Test-english }\end{array}$ & $\begin{array}{ll} & \text { english } \\
\text { - } & \text { English } \\
& \text { Digital } \\
& \text { Academy } \\
\text { - } & \text { Open english } \\
\text { - } & \text { Quick } \\
& \text { learning } \\
\text { - } & \text { Tandem } \\
\text { - } & \text { You talk TV }\end{array}$ & $\begin{array}{ll}\text { - } & \text { Netflix } \\
\text { - } & \text { Youtube } \\
\text { - } & \text { Zoom }\end{array}$ \\
\hline
\end{tabular}

Tabla 1. Sitios y medios utilizados para el estudio autónomo.

El estudio autónomo del inglés durante la pandemia por COVID-19, es una actividad que la mayoría de los estudiantes disfruta llevar a cabo, con una amplia aceptación del 48\%; no obstante, hay un $7 \%$ que indicó disfrutar poco este tipo de estudio.

El dominio de la lengua inglesa por parte de los estudiantes de la LLE es en su mayoría nivel B1 intermedio, un número más reducido tiene nivel B2 intermedio alto. Únicamente un estudiante considera tener el nivel $\mathrm{C} 1$ avanzado en lengua inglesa. A pesar de establecer un buen nivel de dominio del inglés la mayoría de los participantes; existe un pequeño grupo de estudiantes que considera tener dominio bajo de la lengua (Gráfica 9).

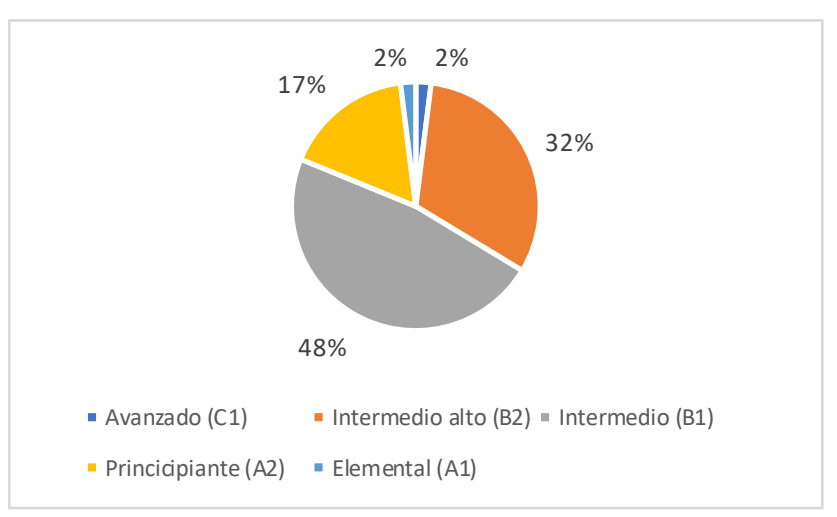

Gráfica 9. Dominio de la lengua inglesa de los estudiantes LLE.

El tiempo de estudio de la lengua inglesa a lo largo de la vida de los estudiantes, supera los dos años en el $97 \%$ de ellos; el $3 \%$ restante tiene un año o menos estudiando lengua inglesa, lo que indica que su primer contacto con el idioma fue a su ingreso a la Facultad de Lengua UAEMex.

\section{CONCLUSIONES}


Las actividades académicas en todo México se vieron afectadas por la COVID-19, situación que no se previó a pesar de la tendencia mundial en lo referente a este coronavirus. Particularmente, la enseñanza del inglés en la Facultad de Lenguas UAEMex no se ha visto afectada, ya que el 100\% de los participantes cuenta con la tecnología necesaria para atender las clases y realizar las tareas.

El autoaprendizaje de la lengua inglesa, durante la pandemia COVID-19, es practicado por más del $70 \%$ de los estudiantes, lo que comprueba que los estudiantes utilizan el internet para realizar estudio del inglés de manera autónoma durante la contingencia sanitaria. Sin embargo, un porcentaje de estudiantes no pudo llevar a cabo esta actividad por la falta de conectividad a internet en sus hogares; lo que conlleva a buscar estrategias alternas al internet para el estudio autónomo del inglés en posibles contextos de contingencia a futuro.

Los estudiantes han desarrollado la competencia digital de manera experimentada a pesar de no contar siempre con las TIC necesarias para ello. El dominio de la computadora, el teléfono inteligente y la búsqueda de la información en la Web, permiten concluir que los estudiantes son capaces de buscar, almacenar, producir e intercambiar información necesaria para su desarrollo académico profesional de manera autónoma.

\section{REFERENCIAS}

ALTE. (2018). Welcome to alte, the Association of Language Testers in Europe. Obtenido de http://www.alte.org

Barrera, A. (18 de marzo de 2019). Aviso. [Suspensión de clases COVID 19]. Obtenido de https://twitter.com/Rector_UAEM/status/1240494515875721217/p hoto/1

Bennett, S., Maton, K., \& Kervin, L. (2008). The 'digital natives' debate: A critical review of the evidence. British Journal of Educational Technology, 775-786.

Cabero, J. (2015). Reflexiones educativas sobre las tecnologías de la información y la comunicación TIC. CEF, 19-27. Obtenido de https://campusenlinea.reduaz.mx/mtie2/pluginfile.php/3912/mod_ page/content/11/Reflexiones\%20educativas.pdf

CCAES. (2 de junio de 2020). INFORMACIÓN CIENTÍFICATÉCNICA. Enfermedad por coronavirus, COVID-19. Obtenido de https://www.mscbs.gob.es/profesionales/saludPublica/ccayes/alert asActual/nCov-China/documentos/ITCoronavirus.pdf

Chandrasena, M. (2019). Lack of Digital Competence: The Hump in A University - English For Specific Purpose - Classroom. INTERNATIONAL JOURNAL OF SCIENTIFIC \& TECHNOLOGY RESEARCH, 10(8), 948-956.
Cobo, C. (2009). El concepto de tecnologías de la información. Benchmarking sobre las definiciones de las TIC en la sociedad del conocimiento. . ZER, 295-318.

Ferrari, A. (2013). DIGCOMP: A Framework for developing and understanding digital competence in Europe. Luxemburgo: Publications Office of the European Union.

Harmer, J. (2015). The Practice of English Language Teaching (5th ed.). Essex: Pearson Education Limited.

Liu, M., Traphagan, T., Huh, J., Ihn Koh, Y., Choi, G., \& McGregor, A. (2008). Designing Websites for ESL Learners: A Usability Testing Study. CALICO Journal, 207-240.

Morze, N., Smyrnova-Trybulska, E., \& Boiko, M. (2019). The impact of educational trends on the digital competence of students in Ukraine and Poland. En E. Smyrnova-Trybulska, E-learning and STEM Education (págs. 365-379). Katowice-Cieszyn: Studio Noa for University of Silesia.

OJEU. (2006). RECOMMENDATION OF THE EUROPEAN PARLIAMENT AND OF THE COUNCIL of 18 December 2006 on key competences for lifelong learning. Luxembourg: European Union.

Organización Mundial de la Salud. (2020). Coronavirus disease (COVID-19) pandemic. Obtenido de http://www.euro.who.int/en/health-topics/healthemergencies/coronavirus-covid-19/novel-coronavirus-2019-ncov

Organización Panamericana de la Salud. (2020). Informes de Situación para COVID-19. Obtenido de https://www.paho.org/es/tag/informes-situacion-para-covid-19

Pourhosein Gilakjani, A., \& Sabouri, N. (2014). Role of Iranian EFL teachers about using Pronunciation Power Software in the instruction of English pronunciation. English Language Teaching, 139-148.

Prensky, M. (2011). Enseñar a nativos digitales. México: Ediciones SM.

SEP. (14 de marzo de 2020). Comunicado conjunto No. 3 Presentan Salud y SEP medidas de prevención para el sector educativo nacional por COVID-19. Obtenido de https://www.gob.mx/sep/es/articulos/comunicado-conjunto-no-3presentan-salud-y-sep-medidas-de-prevencion-para-el-sectoreducativo-nacional-por-covid-19?idiom=es

Tello, E. (2007). Las tecnologías de la información y comunicaciones (TIC) y la brecha digital: su impacto en la sociedad de México. Revista de Universidad y Sociedad del Conocimiento, 1-8.

UAEM. (2009). Currículum de la Licenciatura en Lengua. Toluca: UAEM - CIGOME.

UNESCO. (2018). A Global Framework of Reference on Digital Literacy Skills for Indicator 4.4.2. Montreal: UNESCO.

Vasan, N. (2020). ICT as an Effective Tool for English Language Teaching and Learning. Studies in Indian Place Names, 40(57), 63-66.

Internacionales de investigación sobre personas con discapacidad. Salamanca; INICO; 2012: 1-12. 
Millaruelo-Trillo JM. Importancia de la implicación del paciente en el autocontrol de su enfermedad: paciente experto. Importancia de las nuevas tecnologías como soporte al paciente autónomo. Aten. Primaria 2010; 42(1): 41-47.

Bonal-Ruiz R, Cascaret-Soto X. Reflexión y debate ¿Automanejo, autocuidado o autocontrol en enfermedades crónicas? Acercamiento a su análisis e interpretación. MEDISAN. 2009; 13(1): 1-10.

Lorig K, Ritter PL, Villa F, Piette JD. Spanish diabetes selfmanagement with and without automated telephone reinforcement. Diabetes Care 2008; 31(3): 408-14.

Kidney Disease Improved Global Outcomes (KDIGO). KDIGO 2012 Clinical Practice Guideline for the Evaluation and Management of Chronic Kidney Disease. Off. J. Int. Soc. Nephrol. 2013; 3(1): 1163. 